\title{
Ambientes Inteligentes en Contextos Educativos: Modelo y Arquitectura
}

\author{
José Guillermo Hernández-Calderón, Edgard Benítez-Guerrero, \\ Carmen Mezura-Godoy \\ Facultad de Estadística e Informática, Universidad Veracruzana, Xalapa, México \\ \{guillermohernandez02, edbenitez, cmezura\}@uv.mx
}

\begin{abstract}
Resumen. La Inteligencia Ambiental (Ambient Intelligence o AmI) es un área de la Computación que se dirige a tener espacios, tecnológicamente enriquecidos, que proactivamente apoyen a las personas en su vida diaria. Dada la riqueza de información y conocimientos existentes en ámbitos educativos, la AmI puede proveer soluciones que se adapten a las necesidades y generen beneficios para los usuarios de estos entornos. Este artículo propone un modelo de ambientes educativos inteligentes, así como una arquitectura conceptual para sistemas que soporten dicho modelo.
\end{abstract}

Palabras Clave: Inteligencia Ambiental, Ambientes Educativos Inteligentes, Cómputo Consciente del Contexto

\section{$1 \quad$ Introducción}

La Inteligencia Ambiental (Ambient Intelligence o AmI, en Inglés) es un área de la Computación que se dirige a tener espacios, tecnológicamente enriquecidos, que proactivamente apoyen a las personas en su vida diaria [1]. Entre las principales características que se presentan en los sistemas de AmI se encuentran poder detectar información del entorno, razonar con los datos acumulados y seleccionar las acciones a tomar con el fin de beneficiar a sus usuarios [1]. Entre los campos de aplicación encontrados, destacan los siguientes: casas inteligentes, servicios de salud (hospitales, asistencia, servicios de emergencia), espacios de trabajo y entornos educativos. Es en este último campo de aplicación donde se enfoca este trabajo.

Dada la riqueza de información y de conocimientos existentes en ámbitos educativos, la inteligencia ambiental puede proveer soluciones que se adapten a las necesidades de los diferentes tipos de usuarios de estos entornos (estudiantes, profesores, personal administrativo), permitiendo facilitar sus actividades diarias a través del análisis de la información existente usando nuevas tecnologías y enfoques. Considere el siguiente escenario: 
Juan es un estudiante del $6^{\circ}$ semestre de la licenciatura en Derecho en el Sistema de Enseñanza Abierta de la Universidad Veracruzana. Para estudiar, Juan asiste a un aula inteligente. Antes de que Juan llegue al aula, ésta recupera su información académica (historial académico, horario, estilo de aprendizaje) e identifica, mediante su análisis, actividades y recursos educativos que puedan ser de utilidad para Juan. El aula contacta local o remotamente al profesor del curso para informarle el resultado del análisis y éste le indica si está de acuerdo con ello (en caso de no estar, el profesor puede modificar lo que considere necesario). Una vez que Juan llega al aula, ésta lo identifica y le asigna las actividades preparadas. Conforme Juan va realizando las actividades, el aula monitorea su desempeño para verificar si es el adecuado y, si no, le propone materiales suplementarios personalizados.

Contar con un aula inteligente como la descrita en el escenario anterior no es sencillo. Existen trabajos que han abordado el tema de la inteligencia ambiental en entornos educativos desde diversos ángulos, como el reconocimiento de los usuarios y el desarrollo de actividades dentro del aula mediante dispositivos aumentados que se encargan de recolectar información para mostrarla al maestro. De igual manera hay avances en la interconexión de diversos dispositivos que permiten al maestro facilitar el modo de impartir su clase y grabarla, así como para ofrecer a los alumnos la posibilidad de asistir remotamente a la clase. No obstante existen limitaciones, como que no consideran los aspectos que rodean al usuario y al entorno educativo al realizar la actividad (el contexto); tampoco contemplan la información académica del usuario o sus preferencias para realizar sugerencias al maestro. Nuestro trabajo se dirige a proponer una solución a esta problemática.

El objetivo de este artículo es doble. Por un lado, propone un modelo de ambientes educativos inteligentes que considera los elementos encontrados en un entorno educativo así como aspectos de cómputo centrado en el usuario y, por otro, una arquitectura conceptual de sistema que soporte el modelo.

El resto de este documento está organizado de la siguiente manera. Primero se describen aplicaciones existentes de inteligencia ambiental en entornos educativos y se hace su análisis considerando el modelado, la detección y el uso de la información del ambiente. Después se presenta una propuesta de modelo para ambientes educativos inteligentes y posteriormente se introduce una propuesta de arquitectura. Finalmente se presentan las conclusiones y el trabajo futuro.

\section{Ambientes inteligentes en entornos educativos}

\subsection{Aplicaciones y sistemas}

En la actualidad se pueden encontrar diversos trabajos relacionados con el tema de la inteligencia ambiental aplicada a entornos educativos. A continuación se describen algunos de ellos. 
Shi et al [10] describen un salón en el cual se imparten clases que pueden ser atendidas por estudiantes locales y remotos, ofreciéndoles la posibilidad de interactuar como si estuvieran físicamente en él. El profesor y los alumnos que se encuentran de manera local interactúan a través de un pizarrón interactivo y de lápices digitales para hacer anotaciones que los estudiantes remotos también pueden visualizar. La interacción con los estudiantes remotos se da a través de un Student Board en el que se muestra la imagen de los estudiantes conectados y un asistente virtual, que representa al salón inteligente y con el que el maestro pueda interactuar.

Mathioudakis et al [4] presentan un sistema en tiempo real cuyo objetivo es auxiliar al maestro dentro de un salón inteligente. Este sistema monitorea las actividades de los estudiantes para mostrarle al docente información en una student card; entre esta información se encuentra información personal (como nombre y foto de perfil) e información con respecto a las actividades que ha realizado el estudiante durante el curso. El objetivo es brindarle al maestro la información relevante de cada estudiante, de forma que le permita decidir las estrategias de enseñanza a utilizar u ofrecer asesoría.

Songdo City [12] se enfoca en la inclusión de tecnología en el aula, dotando a los alumnos con tabletas y a las aulas con cámaras y pantallas. Se implementan varias ideas de la Inteligencia Ambiental a gran escala, a nivel de una ciudad, para facilitar a los habitantes el acceso a recursos.

AmI-DesK es un dispositivo desarrollado por Antona et al [7]. Se trata de un objeto cotidiano del ambiente (un escritorio) pero aumentado con tecnología para la recolección de datos relacionados al alumno y a la actividad que está realizando, con el fin de generar información útil al maestro para adecuar las estrategias y brindarle recursos al alumno que le permitan mejorar su desempeño durante el curso.

Leonidis et al. [3], [8] proponen ClassMate, un framework abierto para Ambientes Inteligentes en entornos educativos el cual facilita la interoperabilidad de los servicios del ambiente, comunicación síncrona y asíncrona, capacidad de adaptación y seguridad, entre otros. El núcleo de ClassMate consta de cinco componentes: seguridad, perfil del usuario, administrador de dispositivos, espacio de información y un administrador del contexto, el cual se encarga de orquestar las interacciones entre los otros componentes para satisfacer las necesidades de los usuarios. ClassMate ha sido utilizado para realizar la herramienta de ayuda The Teacher Assistant, en la que el docente puede monitorear en tiempo real las actividades que se están realizando en el aula e identificar incidencias que se presenten.

\subsection{Análisis de los sistemas de AmI educativos}

Los trabajos presentados fueron analizados considerando las siguientes dimensiones: modelado, detección y uso de la información del ambiente. Esto con el fin de identificar las ventajas y las limitaciones de los trabajos considerados. A continuación se presenta dicho análisis. 
Modelado del ambiente. El modelado del ambiente comprende a los elementos lógicos y físicos que se encuentran dentro de él. Algunas propuestas como las de Bravo et al [2], [5], Leonidis et al [3], Antona et al [9] y Nava [6] describen dichos elementos de manera explícita mientras que otros no, como Shi et al [10] y AmI-Desk [7].

Leonidis et al. [3] consideran que en un entorno educativo se encuentran diversos elementos: usuarios, que en la ocurrencia son maestros y alumnos, así como artefactos físicos, como un escritorio aumentado con cámaras y sensores, además de dispositivos móviles, con los que los usuarios interactúan al realizar actividades. Shi et al. [10] también consideran a alumnos y maestros como usuarios, incluyen dispositivos biométricos y también elementos propios de un aula como apuntadores, pizarrones y cámaras. Las funciones principales de su sistema son apoyar al maestro al dar la clase, grabar las clases para subirlas a un repositorio y mejorar la experiencia de los alumnos que toman la clase de manera remota.

Antona et al [9], por su parte, identifican a los alumnos como actores principales, mientras que el maestro y el sistema actúan solamente como facilitadores del aprendizaje proporcionando al alumno los materiales adecuados. Se identifican artefactos que facilitan la recolección de datos de las actividades que están realizando sus usuarios, remplazando los tradicionales como pizarrones y escritorios con otros similares aumentados con tecnología, como por ejemplo el escritorio aumentado (AmiDesk) o un pizarrón aumentado. Los alumnos realizan actividades utilizando estos dispositivos y gracias a la información recolectada por ellos el ambiente puede mostrar al maestro un análisis del desempeño del alumno.

Bravo [2] identifica cuatro elementos básicos de información contextual en un ambiente inteligente. El primero es la identidad (Identity awareness) que permite identificar de manera unívoca a cada usuario, haciendo posible entonces acceder a su perfil y a sus necesidades para poder brindarle información/servicios personalizados. El segundo es la ubicación (Location awareness) que además de conocer a los usuarios presentes en el ambiente, permite conocer qué usuarios están próximos a un tablón virtual, con el fin de mostrarles información, tal como actividades, ejercicios y respuestas en el tablón virtual. El tercero es el tiempo (Time awareness) que permite al sistema conocer el horario de clase o el calendario escolar con el fin de enriquecer la información contextual y ayudar al sistema a ofrecer servicios o recursos al usuario de mejor manera. Finalmente, el cuarto elemento son las tareas (Task awareness), que permite conocer las posibles actividades que el usuario quisiera o pudiera realizar dentro del ambiente. Todo este conocimiento puede ser adquirido a través de la solicitud explícita al usuario o deducido de otros elementos considerados en el contexto.

Detección del ambiente. La identificación de usuarios se ha realizado mediante diversas tecnologías como RFID [5], NFC [6] o sistemas biométricos [10]. Tanto RFID y NFC están basadas en radiofrecuencia, siendo la diferencia entre estas dos tecnologías que RFID solamente funciona como mecanismo de lectura de información previamente almacenada en etiquetas de radiofrecuencia, mientras que en el caso de NFC se añade la fun- 
cionalidad de guardar en las etiquetas información relacionada a eventos que ocurren en el ambiente, misma que podría ser consultada por los usuarios. En lo que respecta a los sistemas biométricos, en The Smart Classroom se utilizan para el reconocimiento del maestro.

Para la recopilación de información relacionada a la actividad que el alumno está realizando, se han ocupado dispositivos aumentados como AmIDesk [7] o también elementos propios del ambiente como los apuntadores, el pizarrón y las cámaras encontrados en The Smart Classroom [10] y con los cuales se puede identificar el espacio del aula donde es llevada a cabo la actividad principal de la clase.

Uso de la información del ambiente. Los elementos identificados por Bravo [2] (identidad, ubicación, tiempo, tarea) son utilizados para ofrecerle al usuario recursos, servicios e información de manera implícita, en otras palabras, sin que éste los solicite explícitamente. Por otro lado, al conocer el lugar donde se está llevando a cabo la actividad principal de la clase a través de los apuntadores y el pizarrón, el sistema en The Smart Classroom es capaz de apuntar las cámaras al lugar principal y poder grabarlo. Por su lado, AmI-RIA [4] utiliza los datos recolectados en las interacciones para identificar situaciones en las que el alumno presente alguna dificultad y para mostrar dicha información al maestro. Por último, el AmIDesk permite al alumno resolver actividades educativas y generar información que será presentada al docente para darle seguimiento, así como materiales multimedia que le pueden ser mostrados como recursos de apoyo.

\section{Modelo de ambiente educativo inteligente}

Esta sección propone un modelo de ambiente educativo inteligente. Para describirlo, hemos adoptado el modelo MARS para aplicaciones groupware [11], ya que considera elementos que también se encuentran en ambientes educativos inteligentes.

Tabla1. Modelo instanciado al caso de estudio

\begin{tabular}{ll}
\hline \multicolumn{1}{c}{ Elemento } & \multicolumn{1}{c}{ Instancia(s) } \\
\hline Arena & "Aula Inteligente" \\
Actores & Juan, Salón 601, Dr. Benítez \\
Objetos & $\begin{array}{l}\text { IntroduccionAlDerecho.pptx, } \\
\text { Primer_examen_parcial.pdf }\end{array}$ \\
Familias de actores y objetos & Estudiante, Salón, Profesor, Material didáctico \\
Modelos de interacción & Seleccionar materiales educativos, \\
& Evaluar sugerencias del aula inteligente, \\
\hline
\end{tabular}




\begin{tabular}{ll}
\hline & $\begin{array}{l}\text { Asignar actividades de aprendizaje, } \\
\text { Realizar actividades de aprendizaje, } \\
\text { Evaluar el desempeño del estudiante }\end{array}$ \\
Roles & $\begin{array}{l}\text { Material Seleccionado, Material Asignado, Evaluador, } \\
\text { Estudiante evaluado }\end{array}$ \\
Guiones & $\begin{array}{l}\text { Seleccionar materiales educativos de acuerdo al perfil } \\
\text { del estudiante y al contexto de aprendizaje } \\
\text { Asignar actividades de aprendizaje de acuerdo a los } \\
\text { recursos disponibles (mensaje de texto, audio) }\end{array}$ \\
\hline
\end{tabular}

En MARS una actividad colaborativa se desarrolla dentro de un espacio denominado Arena. En ella participan actores realizando interacciones reguladas de acuerdo a un modelo de interacción. Los actores en las interacciones manipulan y producen objetos siguiendo guiones que describen cómo se realizan las interacciones. Los actores y los objetos asumen roles durante las interacciones.

La Tabla 1 muestra la instanciación de MARS con los elementos del caso de estudio presentado en la Introducción, en el que el ambiente personaliza los contenidos de acuerdo a las características del usuario. Cabe destacar que en dicho escenario el ambiente funciona como un asistente del maestro que le muestra sugerencias de acuerdo a las características del usuario, al entorno y a la actividad.

\section{$4 \quad$ Arquitectura conceptual}

Esta sección propone una arquitectura conceptual para sistemas de AmI en aulas inteligentes. Dicha arquitectura soporta el modelo presentado en la sección precedente y se espera sea la base para posibles realizaciones concretas. La arquitectura (ver Figura 1) se divide en cuatro capas que son: capa de elementos físicos y lógicos propios del entorno, capa de reconocimiento y acción, capa de análisis y capa de datos.

\subsection{Elementos físicos y lógicos del entorno}

La primera capa engloba a los elementos físicos y lógicos que se encuentran dentro del ambiente y pueden ser utilizados por el usuario o el sistema para interactuar y realizar una actividad. Ejemplos de estos son: pizarrón interactivo o inteligente, aplicaciones educativas, materiales digitales, teléfonos móviles, tarjetas de identificación, cámaras, entre otros. Además de ser utilizados para realizar una actividad, estos elementos permiten la recolección de datos por parte del sistema para conocer al usuario y las posibles actividades que se encuentra realizando a través de la capa de reconocimiento y acción. 


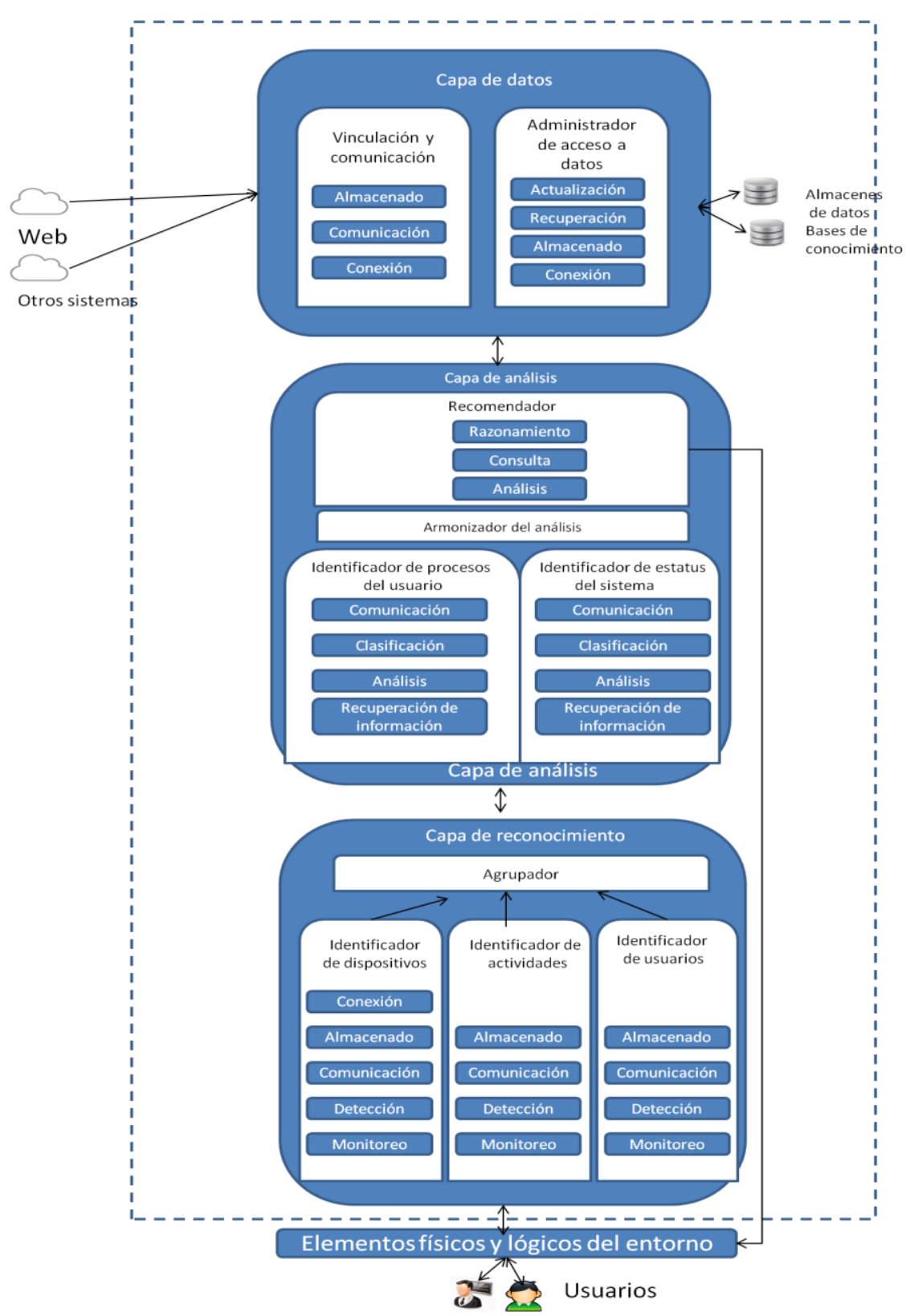

Fig. 1 Arquitectura conceptual. 


\subsection{Capa de reconocimiento}

La capa de reconocimiento engloba todos los componentes necesarios para que el sistema pueda identificar todo lo que se encuentra dentro del entorno educativo.

Identificador de dispositivos. Su función es la de identificar los dispositivos físicos dentro del ambiente, donde dispositivo se refiere a cualquier herramienta de trabajo o comunicación dentro del aula que tenga la posibilidad de recibir o transmitir datos y conectarse al sistema.

Este componente, además de identificarlos, debe clasificarlos para poder relacionarlos mejor de acuerdo a su función, su tipo y su modo de comunicación. También debe ser capaz de identificar y poder establecer canales de comunicación con los dispositivos que se conecten por periodos cortos de tiempo, como los teléfonos móviles de los usuarios.

Identificador del usuario. Su función es la identificación del usuario y la recuperación de la información básica relacionada con él, de acuerdo al momento en que el usuario se encuentra en el ambiente.

Identificador de actividades. Monitorea los eventos del entorno, a través de los dispositivos disponibles, para identificar posibles actividades que se están llevando a cabo.

Agrupador. Su función es recolectar/recibir la información generada por los componentes de la capa de reconocimiento, estructurarla y enviarla a la capa de análisis. Sirve como un intermediario de comunicación entre los componentes de la capa de reconocimiento y la capa de análisis.

\subsection{Capa de análisis}

La capa de análisis de datos se dirige a determinar la situación actual del usuario y del ambiente de acuerdo a la información generada en la capa anterior, sin dejar de lado la consulta del histórico de datos almacenados por el sistema, las reglas propias de la Arena o espacio en el que se llevan a cabo las actividades, así como las características del usuario.

Identificador de procesos del usuario. Este componente determina la situación actual del usuario dentro del entorno. Un ejemplo de ello es: "El usuario Juan cursa el 6to semestre y su promedio es de 8.6, puede tener problemas con la materia de Derecho Privado 
Romano y se encuentra realizando actividades para reforzar el tema Inicios del Derecho Privado".

Identificador de estatus del sistema. Determina el estado actual del sistema y del ambiente en el momento que se lleva a cabo la actividad. Una posible salida de este componente es "Se tienen 5 recursos disponibles relacionados con inicios del derecho privado, la temperatura actual del entorno es de $34^{\circ}$, hay un teléfono móvil perteneciente a Juan conectado, una computadora con dirección Mac e IP de 00 8A 01 3E 00 y 192.168.1.2, un cañón conectado y otros dispositivos de comunicación, son las 14:32 horas y las actividades actuales están programadas para terminar a las 15 horas."

Armonizador del análisis. Armoniza la información de la situación actual del usuario y del sistema para enviarla al Recomendador. Este componente tiene como función principal estructurar y clasificar la información analizada para realizar los procesos necesarios en el Recomendador que permitan sugerir las mejores opciones de acuerdo al material disponible y a las características del usuario y el entorno en el cual se está llevando a cabo la actividad.

Recomendador. Este componente se encarga de sugerir, de acuerdo a la información obtenida y analizada, el contenido educativo que mejor se adapte a la situación actual del usuario. Esta sugerencia es enviada al maestro para que la evalúe y determine si el contenido sugerido se muestre finalmente al alumno o si se debe cambiar por otro.

\subsection{Capa de datos}

La capa de datos contempla la información que es generada por los elementos del ambiente y que es consultada por el ambiente para personalizar y hacer sugerencias a los usuarios. Está compuesta de 3 elementos: almacenes de datos, acceso a datos y por último el de comunicación. Los almacenes de datos contienen toda la información generada por el sistema y se consideran: (1) bases de datos para almacenar la información relacionada a usuarios, actividades, estados del entorno y el histórico de la información generada en el ambiente, (2) bases de conocimiento que contienen las reglas que definirán el comportamiento del sistema de acuerdo a actividades y hechos registrados previamente en el sistema y modelos de usuario y ambiente, que definen las características básicas de usuario y ambiente que permitan clasificar y actuar de mejor manera. Por su parte, el acceso a datos se refiere a servicios que permitan recuperar la información almacenada en las base de datos y en las bases de conocimiento. Por último está el elemento de comunicación cuya función principal es la de comunicarse para extraer información relacionada al usuario 
que pudiera no estar almacenada directamente en el sistema pero sí en la web o en otros sistemas.

\section{Conclusiones y Trabajo Futuro}

Este artículo propone un modelo así como una arquitectura funcional para sistemas de AmI, particularmente para aquellos orientados a entornos educativos. El modelo propuesto considera los elementos fundamentales que se encuentran en este tipo de sistemas (actores, tales como maestros y alumnos, sus actividades, así como los objetos que manipulan). La arquitectura conceptual, por su lado, propone componentes abstractos que soportan los elementos del modelo.

Como trabajo futuro se considera la validación experimental tanto del modelo como de la arquitectura propuestos, mediante el desarrollo y uso de un prototipo de entorno educativo inteligente. Se espera que los resultados que se obtengan permitirán identificar áreas de oportunidad para la mejora de las propuestas presentadas en este documento.

\section{Referencias}

1. Cook, D.J., Augusto, J.C., Jakkula, V.R. Review: Ambient intelligence: Technologies, applications, and opportunities. Pervasive and Mobile Computing 5, 277-298 (2009).

2. Bravo, J., Hervás, R., Sánchez, I., Crespo, A. U. Servicios por identificación en el aula ubicua. En VI Simposio internacional de Informática Educativa (SIIE'04), pp. $26-27$ (2004)

3. Leonidis, A., Margetis, G., Antona, M., Stephanidis, C. ClassMATE: Enabling Ambient Intelligence in the Classroom. World Academy of Science, Engineering and Technology, 66, 594598 (2010)

4. Mathioudakis, G., Leonidis, A., Korozi, M., Margetis, G., Ntoa, S., Antona, M., Stephanidis, C. (2013). AmI-RIA: Real-Time Teacher Assistance Tool for an Ambient Intelligence Classroom, En Proceedings of the Fifth International Conference on Mobile, Hybrid, and On-Line Lerning (eLmL 2013), 37-42 (2013)

5. Bravo, J., Hervás, R., Chavira, G. Ubiquitous Computing in the Classroom: An Approach through Identification Process. Journal of Universal Computer Science, 11(9), 1494-1504 (2005).

6. Nava, S. Modelado de un ambiente inteligente: Un entorno consciente del contexto a través del etiquetado. Tesis Doctoral. Escuela Superior de Informática, Universidad de Castilla - La Mancha (2010)

7. Antona, M., Margetis, G., Ntoa, S., Leonidis, A., Korozi, M., Paparoulis, G., Stephanidis, C. Ambient Intelligence in the Classroom: an Augmented School Desk. En Proceedings of the 3rd International Conference on Applied Human Factors and Ergonomics (2010)

8. Leonidis, A., Korozi, M., Margetis, G., Ntoa, S., Papagiannakis, H., \& Stephanidis, C. A Glimpse into the Ambient Classroom, Bulletin of the IEEE Technical Committee on Learning Technology, 14(4), 3 (2012) 
9. Antona, M., Leonidis, A., Margetis, G., Korozi, M., Ntoa, S., \& Stephanidis, C. A StudentCentric Intelligent Classroom, En Proceedings of the Second International Joint Conference on AmI, 248-252 (2011).

10. Shi, Y., Xie, W., Xu, G., Shi, R., Chen, E., Mao, Y., \& Liu, F. The Smart Classroom: Merging Technologies for Seamless Tele-Education. IEEE Pervasive Computing, 2(2), 47-55 (2003)

11. Mezura-Godoy, C, Talbot, S. Towards Social Regulation in Computer-Supported Collaborative Work. En Proceedings of the 9th International Workshop on Groupware: Design, Implementation, and Use (CRIWG 2003), pp 84-89 (2003).

12. Schiller, A., \& Park, M. The International Campus as Prototype for International Collaboration: South Korea's Incheon Global Campus. Global Partners in Education Journal, 4(1), 33-40 (2014) 